\title{
Vertex Domination on S - Valued Graphs
}

\author{
S.Jeyalakshmi*And M.Chandramouleeswaran** \\ *Devanga Arts College Aruppukottai - 626101. Tamilnadu. India. \\ ${ }^{*}$ Saiva Bhanu Kshatriya College Aruppukottai - 626101. Tamilnadu. India.
}

\begin{abstract}
In [5], we introduced the notion of $S^{-}$valued graphs, where $S$ is a semiring. The fastest growing area in graph theory is the study of domination. In this paper, we introduce the notion of Vertex domination on $S$ - valued graphs and prove simple properties.
\end{abstract}

Keywords: Semirings, Graphs, $S$-valued graphs, Vertex Dominating set.

AMS Classification: 05C25, $16 Y 60$

\section{Introduction}

H.S. Vandiver [7], in the year 1934, introduced the notion of semirings, while studying the algebraic structure of ideals in rings. In [2], Jonathan Golan introduced the notion of $\mathrm{S}-$ valued graphs, where $\mathrm{S}$ is a semiring. However, nothing more have been said. This motivated us to study the notion of semiring valued graphs (simply called the S-valued graphs) in our paper [5].

The fastest growing area within Graph theory is the study of domination, the reason being its many and varied applications in such fields as social sciences, communications, networks, algorithmic designs etc. The topic of domination was given formal Mathematical definition by [1] Berge in 1958. He introduced the coefficient of external stability, which is now known as the domination number of a graph. [4]Ore introduced the terms dominating set and domination number in the year 1962. Motivated by the domination theory in graphs, in this paper, we introduce the notion of Vertex Domination on S- valued graphs and discuss some simple but elegant results.

\section{Preliminaries}

In this section, we recall some basic definitions that are needed for our work.

Definition 2.1. [2] A semiring $(\mathrm{S},+, \cdot)$ is an algebraic system with a non-empty set $\mathrm{S}$ together with two binary operations + and $\cdot$ such that

(1) $\quad(\mathrm{S},+, 0)$ is a monoid.

(2) $\quad(\mathrm{S}, \cdot)$ is a semi group.

(3) For all a,b,c $\in \mathrm{S}, \mathrm{a} \cdot(\mathrm{b}+\mathrm{c})=\mathrm{a} \cdot \mathrm{b}+\mathrm{a} \cdot \mathrm{c}$ and $(\mathrm{a}+\mathrm{b}) \cdot \mathrm{c}=\mathrm{a} \cdot \mathrm{c}+\mathrm{b} \cdot \mathrm{c}$.

(4) $0 \cdot \mathrm{x}=\mathrm{x} \cdot 0=0 \quad \forall \mathrm{x} \in \mathrm{S}$.

Definition 2.2. Let $(\mathrm{S},+, \cdot)$ be a semiring. A Canonical Pre-order $\lesssim$ in $S$ defined as follows: for $\mathrm{a}, \mathrm{b} \in \mathrm{S}$, a $\mathrm{b}$ if, there exists $\mathrm{c} \in \mathrm{S}$ such that $\mathrm{a}+\mathrm{c}=\mathrm{b}$.

Definition 2.3. A set $D \subseteq V$ of vertices in a graph $G=(V, E)$ is called a Dominating vertex set in $G$ if every vertex $v \in \mathrm{V}$ is either an element in $\mathrm{D}$ or is adjacent to an element in $\mathrm{V}-\mathrm{D}$.

A set $\mathrm{D} \subseteq \mathrm{V}$ is a Dominating vertex set of $\mathrm{G}$, if $\forall \mathrm{v} \in \mathrm{V}-\mathrm{D}, \mathrm{N}(\mathrm{v}) \cap \mathrm{D} \neq \phi$.

Definition 2.4. A dominating set $\mathrm{D}$ is a minimal dominating vertex set if no proper subset of $\mathrm{D}$ is a vertex dominating set in $\mathrm{G}$.

Definition 2.5. A set $D \subseteq V$ is a minimal dominating set of the graph $G=(V, E)$ if $D$ is a dominating set and $\forall \mathrm{v} \in \mathrm{D}$, either $\mathrm{v}$ has no neighbour in $\mathrm{D}$ or there exists neighbour $\mathrm{u} \in \mathrm{V}-\mathrm{D}$ of $\mathrm{v}$ such that $\mathrm{u}$ has no neighbor in $\mathrm{D} \backslash\{\mathrm{v}\}$.

Definition 2.6. A set $D \subseteq V$ is an Independent set of $G$ if $u, v \in D, N(u) \cap\{v\}=\varphi$.

Definition 2.7. A set $D \subseteq V$ is an Independent dominating set of $G$ if $D$ is both an independent and a dominating set.

Definition 2.8. [5]

Let $\mathrm{G}=(\mathrm{V}, \mathrm{E} \subset \mathrm{V} \times \mathrm{V})$ be a given graph with $\mathrm{V}, \mathrm{E} \neq \varphi$. For any semiring $(\mathrm{S},+, \cdot)$, a semiring-valued graph (or a $\mathrm{S}$-valued graph), $\mathrm{G}^{\mathrm{S}}$, is defined to be the graph $\mathrm{G}^{\mathrm{S}}=(\mathrm{V}, \mathrm{E}, \sigma, \psi)$ where $\sigma: \mathrm{V} \rightarrow \mathrm{S}$ and $\psi: \mathrm{E} \rightarrow \mathrm{S}$ is defined to be

$$
\psi(x, y)=\left\{\begin{array}{cc}
\min \{\sigma(x), \sigma(y)\} & \text { if } \sigma(x) \preceq \sigma(y) \text { or } \sigma(y) \preceq \sigma(x) \\
0 & \text { otherwise }
\end{array}\right.
$$


for every unordered pair $(\mathrm{x}, \mathrm{y})$ of $\mathrm{E} \subset \mathrm{V} \times \mathrm{V}$. We call $\sigma$, a $\mathrm{S}-\mathrm{vertex}$ set and $\psi$, a $\mathrm{S}-$ edge set of $\mathrm{G}^{\mathrm{S}}$.

Definition 2.9. Consider the $S-$ valued graph $G^{S}=(V, E \subset V \times V, \sigma, \psi)$. The open neighbourhood of $v_{i}$ in $G^{S}$ is defined as the set $\mathrm{N}_{\mathrm{S}}\left(\mathrm{v}_{\mathrm{i}}\right)=\left\{\left(\mathrm{v}_{\mathrm{j}}, \sigma\left(\mathrm{v}_{\mathrm{j}}\right)\right)\right.$, where $\left.\left.\left(\mathrm{v}_{\mathrm{i}}, \mathrm{v}_{\mathrm{j}}\right) \in \mathrm{E}, \psi\left(\mathrm{v}_{\mathrm{i}}, \mathrm{v}_{\mathrm{j}}\right) \in \mathrm{S}.\right)\right\}$

Definition 2.10. The closed neighbourhood of $v_{i}$ in $G^{S}$ is defined to be the set

$\mathrm{N}_{\mathrm{S}}\left[\mathrm{v}_{\mathrm{i}}\right]=\mathrm{N}_{\mathrm{S}}\left(\mathrm{v}_{\mathrm{i}}\right) \cup\left\{\left(\mathrm{v}_{\mathrm{i}}, \sigma\left(\mathrm{v}_{\mathrm{i}}\right)\right)\right\}$

Definition 2.11. A vertex $v \in D$ of $G^{S}$ is said to be an $S-$ isolate vertex if $N_{S}(v) \subseteq D^{\prime} \times S$ where $D^{\prime}=V-D^{\prime}$.

Definition 2.12. [6] The Degree of the vertex $v_{i}$ of the $S-v a l u e d$ graph $G^{S}$ is defined as

$\operatorname{deg}_{\mathrm{S}}\left(\mathrm{v}_{\mathrm{i}}\right)=\left(\Sigma_{(\mathrm{vi}, \mathrm{v}) \in \mathrm{E}} \psi\left(\mathrm{v}_{\mathrm{i}}, \mathrm{v}_{\mathrm{j}}\right), \ell\right)$ where $\ell$ is the number of edges incident with $\mathrm{v}_{\mathrm{i}}$.

\section{Vertex Domination on $\mathrm{S} \square$ Valued Graphs}

In this section, we introduce the notion of vertex dominating set and prove some properties.

Definition 3.1. A vertex $\mathrm{v}$ in $\mathrm{G}^{\mathrm{S}}$ is said to be a weight dominating vertex if $\sigma(u) \preceq \sigma(v), \quad \forall u \in N_{S}[v]$.

Example 3.2. Let $(S=\{0, a, b, c\},+, \cdot)$ be a semiring with the following Cayley tables:

\begin{tabular}{|l|l|l|l|l|}
\hline+ & 0 & $\mathrm{a}$ & $\mathrm{b}$ & $\mathrm{c}$ \\
\hline 0 & 0 & $\mathrm{a}$ & $\mathrm{b}$ & $\mathrm{c}$ \\
\hline $\mathrm{a}$ & $\mathrm{a}$ & $\mathrm{a}$ & $\mathrm{b}$ & $\mathrm{c}$ \\
\hline $\mathrm{b}$ & $\mathrm{b}$ & $\mathrm{b}$ & $\mathrm{b}$ & $\mathrm{b}$ \\
\hline $\mathrm{c}$ & $\mathrm{c}$ & $\mathrm{c}$ & $\mathrm{b}$ & $\mathrm{b}$ \\
\hline
\end{tabular}

\begin{tabular}{|l|l|l|l|l|}
\hline$\cdot$ & 0 & $a$ & $b$ & $c$ \\
\hline 0 & 0 & 0 & 0 & 0 \\
\hline a & 0 & 0 & $a$ & 0 \\
\hline$b$ & 0 & a & b & c \\
\hline c & 0 & 0 & c & c \\
\hline
\end{tabular}

let be a canonical pre-order in $S$, given by $0 \preceq 0,0 \preceq a, 0 \preceq b, 0 \preceq c, a \preceq a, a \preceq b, a \preceq c, b \preceq b, c \preceq c, c \preceq b$ Consider the $S$ - valued graph $G^{S}=(V, E, \sigma, \psi)$

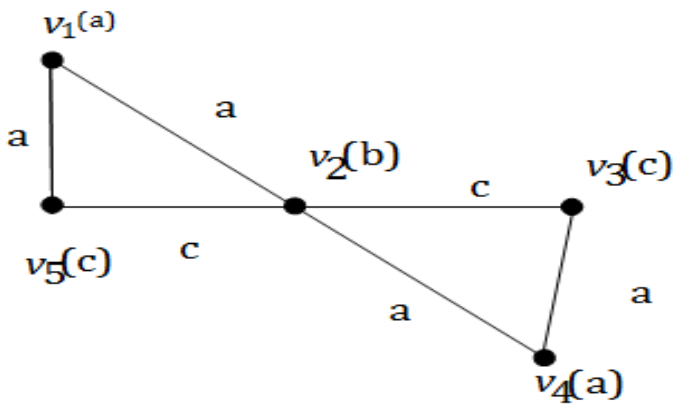

where $\sigma: \mathrm{V} \rightarrow \mathrm{S}$ is defined by

$\sigma\left(\mathrm{v}_{1}\right)=\sigma\left(\mathrm{v}_{4}\right)=\mathrm{a} ; \sigma\left(\mathrm{v}_{2}\right)=\mathrm{b}$ and $\sigma\left(\mathrm{v}_{3}\right)=\sigma\left(\mathrm{v}_{5}\right)=\mathrm{c}$

and $\psi: \mathrm{E} \rightarrow \mathrm{S}$ by

$\psi\left(\mathrm{v}_{1}, \mathrm{v}_{2}\right)=\psi\left(\mathrm{v}_{1}, \mathrm{v}_{5}\right)=\psi\left(\mathrm{v}_{2}, \mathrm{v}_{4}\right)=\psi\left(\mathrm{v}_{3}, \mathrm{v}_{4}\right)=\mathrm{a}$ and $\psi(\mathrm{v} 2, \mathrm{v} 3)=\psi(\mathrm{v} 2, \mathrm{v} 5)=\mathrm{c}$

In graph $\mathrm{G}^{\mathrm{S}}, \mathrm{v}_{2}$ is a weight dominating vertex.

Definition 3.3. A subset $\mathrm{D} \subseteq \mathrm{V}$ is said to be a weight dominating vertex set if for each $v \in D, \sigma(u) \preceq \sigma(v), \quad \forall \quad u \in N_{S}[v]$.

Example 3.4. Consider the semiring $(S=\{0, a, b, c\},+, \cdot)$ with canonical pre-order given in Example 3.2.

Consider the $\mathrm{S}-$ graph $\mathrm{G}^{\mathrm{S}}=(\mathrm{V}, \mathrm{E}, \sigma, \psi)$. 


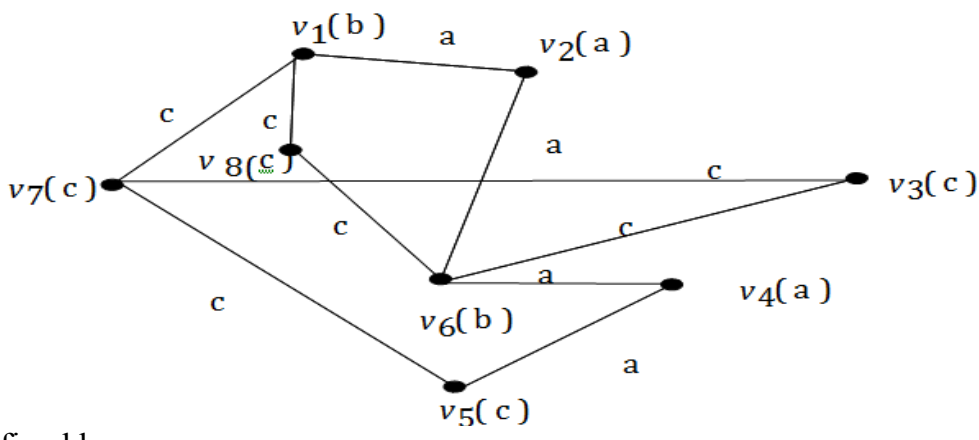

where $\sigma: \mathrm{V} \rightarrow \mathrm{S}$ is defined by

$\sigma\left(\mathrm{v}_{1}\right)=\sigma\left(\mathrm{v}_{6}\right)=\mathrm{b} ; \sigma\left(\mathrm{v}_{2}\right)=\sigma\left(\mathrm{v}_{4}\right)=\mathrm{a}$ and $\sigma\left(\mathrm{v}_{3}\right)=\sigma\left(\mathrm{v}_{5}\right)=\sigma\left(\mathrm{v}_{7}\right)=\sigma\left(\mathrm{v}_{8}\right)=\mathrm{c}$.

and $\psi: \mathrm{E} \rightarrow \mathrm{S}$ by

$\psi\left(\mathrm{v}_{1}, \mathrm{v}_{2}\right)=\psi\left(\mathrm{v}_{2}, \mathrm{v}_{6}\right)=\psi\left(\mathrm{v}_{4}, \mathrm{v}_{6}\right)=\psi\left(\mathrm{v}_{4}, \mathrm{v}_{5}\right)=\mathrm{a}$ and

$\psi\left(\mathrm{v}_{1}, \mathrm{v}_{8}\right)=\psi\left(\mathrm{v}_{1}, \mathrm{v}_{7}\right)=\psi\left(\mathrm{v}_{3}, \mathrm{v}_{7}\right)=\psi\left(\mathrm{v}_{3}, \mathrm{v}_{6}\right)=\psi\left(\mathrm{v}_{5}, \mathrm{v}_{7}\right)=\psi\left(\mathrm{v}_{6}, \mathrm{v}_{8}\right)=\mathrm{c}$.

Here, $D=\left\{\mathrm{v}_{1}, \mathrm{v}_{5}, \mathrm{v}_{6}\right\}$ is a weight $\mathrm{S}$-dominating vertex set of $\mathrm{G}^{\mathrm{S}}$.

However, the set $D_{1}=\left\{v_{1}, v_{3}, v_{5}\right\}$ is not a weight dominating vertex set of $G^{S}$.

Definition 3.5. If $\mathrm{D}$ is a weight dominating vertex set of $\mathrm{G}^{\mathrm{S}}$, then the scalar cardinality of $\mathrm{D}$, denoted by $|\mathrm{D}|_{\mathrm{S}}$,

is defined by $|\mathrm{D}|_{\mathrm{S}}=\sum_{v \in D} \sigma(v)$

For the weight dominating vertex set $\mathrm{D}$ of example, $3.4|\mathrm{D}|_{\mathrm{S}}=\mathrm{b}$.

Definition 3.6. A subset $\mathrm{D} \subseteq \mathrm{V}$ is said to be a minimal weight dominating vertex set if

(1) $\mathrm{D}$ is a weight dominating vertex set.

(2) No proper subset of D is a weight dominating vertex set.

Definition 3.7. The cardinality of the minimal weight dominating vertex set $\mathrm{D} \subseteq \mathrm{V}$ is called the vertex domination number of $\mathrm{G}^{\mathrm{S}}$. It is denoted by $\gamma_{\mathrm{v}}{ }^{\mathrm{S}}\left(\mathrm{G}^{\mathrm{S}}\right)$. That is $\gamma_{V}^{S}\left(G^{S}\right)=\left(|D|_{S},|D|\right)$.

Example 3.8. Consider the semiring $(S=\{0, a, b, c\},+, \cdot)$ with canonical pre-order given in Example 3.2. Consider the $\mathrm{S}-$ valued graph $\mathrm{G}^{\mathrm{S}}=(\mathrm{V}, \mathrm{E}, \sigma, \psi)$.

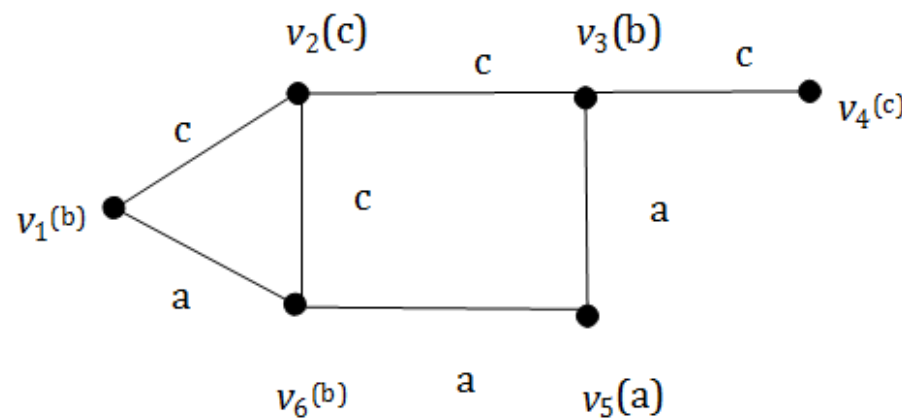

where $\sigma: \mathrm{V} \rightarrow \mathrm{S}$ is defined by

$\sigma\left(\mathrm{v}_{5}\right)=\mathrm{a} ; \sigma\left(\mathrm{v}_{2}\right)=\sigma\left(\mathrm{v}_{4}\right)=\mathrm{c} ;$ and $\sigma\left(\mathrm{v}_{1}\right)=\sigma\left(\mathrm{v}_{3}\right)=\sigma\left(\mathrm{v}_{6}\right)=\mathrm{b}$

and $\psi: \mathrm{E} \rightarrow \mathrm{S}$ by

$\psi\left(\mathrm{v}_{3}, \mathrm{v}_{5}\right)=\psi\left(\mathrm{v}_{5}, \mathrm{v}_{6}\right)=\psi\left(\mathrm{v}_{1}, \mathrm{v}_{6}\right)=\mathrm{a}$ and $\psi\left(\mathrm{v}_{1}, \mathrm{v}_{2}\right)=\psi\left(\mathrm{v}_{2}, \mathrm{v}_{3}\right)=\psi\left(\mathrm{v}_{2}, \mathrm{v}_{6}\right)=\psi\left(\mathrm{v}_{3}, \mathrm{v}_{4}\right)=\mathrm{c}$

Here $D_{1}=\left\{v_{3}, v_{6}\right\}, D_{2}=\left\{v_{1}, v_{3}\right\}, D_{3}=\left\{v_{1}, v_{3}, v_{6}\right\}$ are weight dominating vertex sets of $G^{S}$.

$D_{1}=\left\{v_{3}, v_{6}\right\}$ and $D_{2}=\left\{v_{1}, v_{3}\right\}$ are minimal weight $S-$ dominating vertex sets with

$\left|D_{1}\right|=2=\left|D_{2}\right|$. Further, the scalar cardinality of $D_{1}$ is given by $\left.D_{1}\right|_{s}=\sigma\left(v_{3}\right)+\sigma\left(v_{6}\right)=b+b=b$

and that of $D_{2}$ is given by $\left.D_{2}\right|_{S}=\sigma\left(v_{1}\right)+\sigma\left(v_{3}\right)=a+b=b$ Thus, the domination number of $G^{S}$, is given by $\gamma_{V}^{S}\left(G^{S}\right)=\left(\left|D_{1}\right|_{S},\left|D_{1}\right|\right)=(b, 2)=\left(\left|D_{2}\right| S,\left|D_{2}\right|\right)$

Remark 3.9. Minimal weight $S$-dominating vertex set in a $S$-valued graph need not be, in general, unique, as seen in example 3.8 .

Definition 3.10. A subset $D \subseteq V$ is said to be a maximal weight dominating vertex set if

(1) D is a weight dominating vertex set, and

(2) there is no weight dominating vertex subset $D^{\prime} \subset V$ such that $D^{\prime} \subset D^{\prime} \subset V$.

Example 3.11. Consider the $\operatorname{semiring}(S=\{0, a, b, c\},+, \cdot)$ with canonical preorder given in example 3.2. 
Consider the $S^{-}$-valued graph $G^{S}=(V, E, \sigma, \psi)$.

Where $\sigma: V \rightarrow S$ is defined by

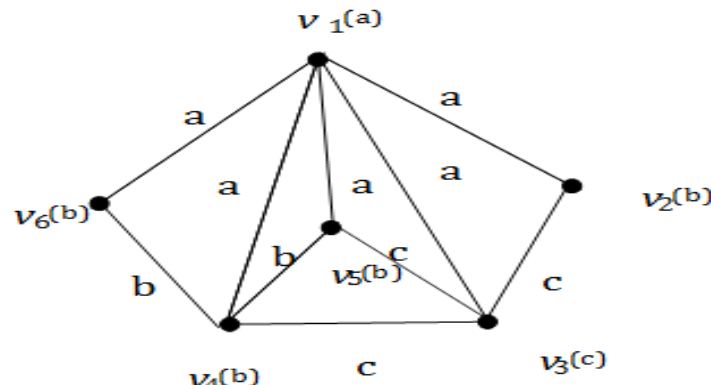

$\sigma\left(v_{1}\right)=a ; \sigma\left(v_{2}\right)=\sigma\left(v_{4}\right)=\sigma\left(v_{5}\right)=\sigma\left(v_{6}\right)=b$ and $\sigma\left(v_{3}\right)=c$

and $\psi: E \rightarrow S$ by

$\psi\left(v_{1}, v_{2}\right)=\psi\left(v_{1}, v_{3}\right)=\psi\left(v_{1}, v_{5}\right)=\psi\left(v_{1}, v_{4}\right)=\psi\left(v_{1}, v_{6}\right)=a ; \psi\left(v_{4}, v_{5}\right)=\psi\left(v_{4}, v_{6}\right)=b$ and

$\psi\left(v_{2}, v_{3}\right)=\psi\left(v_{3}, v_{4}\right)=\psi\left(v_{3}, v_{5}\right)=c$

Here $D_{1}=\left\{v_{2}, v_{4}\right\}, D_{2}=\left\{v_{2}, v_{5}, v_{4}\right\}$ and $D_{3}=\left\{v_{2}, v_{4}, v_{6}\right\}$ are weight dominating vertex sets of $G^{S}$.

$D_{2}=\left\{v_{2}, v_{4}, v_{5}\right\}$ and $D_{3}=\left\{v_{2}, v_{4}, v_{6}\right\}$ are maximal weight dominating vertex sets with $\left|D_{2}\right|=3=\mid D_{3}$

Remark 3.12. Maximal weight $S$-dominating vertex set in a $S$-valued graph, $G^{S}$, need not be, in general, unique.

For, in Example 3.11.

$D_{2}=\left\{v_{2}, v_{5}, v_{4}\right\}$ and $D_{3}=\left\{v_{2}, v_{4}, v_{6}\right\}$ are maximal weight dominating vertex sets with $\left|D_{2}\right|=3=\left|D_{3}\right|$

Theorem 3.13. A weight dominating vertex set $D$ of a graph $G^{S}$ is a minimal weight dominating vertex set of $G$ iff every vertex $v \in D$ satisfies atleast one of the following properties:

(1) there exist a vertex $\mathrm{u} \in \mathrm{V}-\mathrm{D}$ such that $\mathrm{N}_{\mathrm{S}}(\mathrm{u}) \cap(\mathrm{D} \times \mathrm{S})=\{(\mathrm{v}, \sigma(\mathrm{v}))\}$

(2) $\quad v$ is adjacent to no vertex of D.

Proof: Let $\mathrm{v} \in \mathrm{D}$. Assume that $\mathrm{v}$ is adjacent to no vertex of $\mathrm{D}$, then $\mathrm{D}-\{\mathrm{v}\}$ cannot be a weight dominating vertex set. $\Rightarrow \mathrm{D}$ is a minimal weight dominating vertex set.

On the other hand, if for any $\mathrm{v} \in \mathrm{D}$ there exist $\mathrm{a} u \in \mathrm{V}-\mathrm{D}$ such that $\mathrm{N}_{\mathrm{S}}(\mathrm{u}) \cap(\mathrm{D} \times \mathrm{S})=\{(\mathrm{v}, \sigma(\mathrm{v}))\}$

Then $\mathrm{u}$ is adjacent to $\mathrm{v} \in \mathrm{D}$ and no other vertex of $\mathrm{D}$.

In this case also, $\mathrm{D}-\{\mathrm{v}\}$ cannot be a weight dominating vertex set of $\mathrm{G}^{\mathrm{S}}$.

Hence $\mathrm{D}$ is a minimal weight dominating vertex set.

Conversely, assume that $D$ is a minimal weight dominating vertex set of $G^{S}$.

Then for each $\mathrm{v} \in \mathrm{D}, \mathrm{D}-\{\mathrm{v}\}$ is not a dominating set of $\mathrm{G}^{\mathrm{S}}$.

$\therefore$ there exist a $\mathrm{u} \in \mathrm{V}-(\mathrm{D}-\{\mathrm{v}\})$ that is adjacent to no vertex of $(\mathrm{D}-\{\mathrm{v}\})$.

If $\mathrm{u}=\mathrm{v}$, then $\mathrm{v}$ is adjacent to no vertex of $\mathrm{D}$.

If $\mathrm{u} \neq \mathrm{v}$, then $\mathrm{D}$ is a dominating set and $\mathrm{u} \in \mathrm{V}-\mathrm{D} \Rightarrow \mathrm{u}$ is adjacent to atleast one vertex of $\mathrm{D}$.

However $\mathrm{u}$ is not adjacent to any vertex of $\mathrm{D}-\{\mathrm{v}\}$.

$\Rightarrow \mathrm{N}_{\mathrm{S}}(\mathrm{u}) \cap \mathrm{D} \times \mathrm{S}=\{(\mathrm{v}, \sigma(\mathrm{v}))\}$.

Remark 3.14. The above theorem can be rephrased as follows:

A dominating set $D$ of a graph $G^{S}$ is a minimal dominating set of $G^{S}$ iff for every vertex $v \in D$,

(1) either $v$ dominates some vertex of $V-D$ such that no other vertex of $D$ dominates.

(2) or no other vertex of $D$ dominates $v$.

Theorem 3.15. If $D \subseteq V$ is a minimal weight Dominating vertex set $G$ without $S$-isolated vertices then $V-D$ is also a weight dominating vertex set of $G^{S}$.

Proof:

Let $v \in D$. Then by theorem 3.13,

(1) there exist a vertex $u \in V-D$ such that $N_{S}(u) \cap D=\{v\}$

(2) $v$ is adjacent to no vertex of $D$.

In the first case, $v$ is adjacent to some vertex in $V-D$.

In the second case, $v$ is an $S-$ isolated vertex of the subgraph spanned by $\langle D\rangle$.

But $v$ is not $S^{-}$isolated in $G^{S}$.

Hence $v$ is adjacent to some vertex of $V-D$.

Thus $V-D$ is a weight dominating vertex set of $G^{S}$. 
Definition 3.16. A subset $D \subseteq V$ is an Independent set of $G^{S}$ if $u, v \in D, N_{S}(u) \cap\{(v, \sigma(v)\}=\varphi$

Definition 3.17. A subset $D \subseteq V$ is said to be an independent weight dominating vertex set of $G^{S}$ if

(1) D is a weight dominating vertex set.

(2) No two vertices of $D$ are adjacent.

Example 3.18. Consider the semiring $(S=\{0, a, b, c\},+, \cdot)$ with canonical preorder given in example 3.2. Consider the $G^{S}$ :

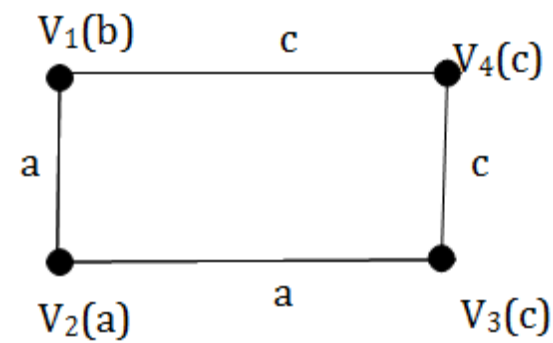

Where $\sigma: V \rightarrow S$ is defined by $\sigma\left(v_{2}\right)=a ; \sigma\left(v_{1}\right)=b$ and $\sigma\left(v_{3}\right)=\sigma\left(v_{4}\right)=c$ and $\psi: E \rightarrow S$ by $\psi\left(v_{1}, v_{2}\right)=\psi\left(v_{2}, v_{3}\right)=a$ and $\psi\left(v_{1}, v_{4}\right)=\psi\left(v_{3}, v_{4}\right)=c$

Here $D=\left\{v_{1}, v_{3}\right\}$ is a weight dominating vertex set of $G^{S}$ and also $v_{1}$ and $v_{3}$ are not adjacent.

$\therefore D$ is an Independent weight dominating vertex set of $G^{S}$. Hence $D=\left\{v_{1}, v_{3}\right\}$ is an Independent set of $G^{S}$.

Theorem 3.19. A set $\mathrm{D} \subseteq \mathrm{V}$ of $\mathrm{G}^{\mathrm{S}}$ is an independent weight dominating vertex set iff $\mathrm{D}$ is a maximal independent set in $\mathrm{G}^{\mathrm{S}}$.

Proof: Clearly every maximal independent set $\mathrm{D}$ in $\mathrm{G}^{\mathrm{S}}$ is a weight dominating vertex set.

Conversely, assume that $\mathrm{D}$ is an independent weight dominating vertex set. Then $\mathrm{D}$ is independent and every vertex not in $D$ is adjacent to a vertex of $D$ and therefore $D$ is a maximal independent set in $G^{S}$.

Theorem 3.20. Every maximal independent set of vertices $D$ in $G^{S}$ is a minimal weight dominating vertex set. Proof : Let $\mathrm{D}$ be a maximal independent set of vertices in $\mathrm{G}^{\mathrm{S}}$. Then by theorem 3.19 , D is a weight dominating vertex set.

Since D is independent, every vertex of D is adjacent to no vertex of D. Thus, every vertex of D satisfies the second condition of theorem 3.13. Hence $D$ is a minimal weight dominating vertex set in $G^{S}$.

\section{References}

[1] Berge C Theory of Graphs and its Applications, Methuen, London, (1962 ).

[2] Jonathan Golan Semirings and Their Applications, Kluwer Academic Publishers, London.

[3] Jeyalakshmi.S, Rajkumar.M, and Chandramouleeswaran.M: Regularity on S- Graphs,(Submitted)

[4] Ore . U: Theory of Graphs, Amer. Math. Soc. Coll09. Publ. 38, (Amer.Math.Soc., Providence RI) 1962.

[5] Rajkumar.M., Jeyalakshmi.S and Chandramouleeswaran.M: Semiring-valued Graphs International Journal of Math. Sci. and Engg. Appls., Vol. 9 (III), 2015, 141152.

[6] Rajkumar.M., and Chandramouleeswaran.M: Degree regular $S$ - valued Graphs, IMRF International Research Journal , Vol. 4 Issue 2 , Kerala (2015).326 - 328.

[7] Vandiver. H.S: Note on a simple type of algebra in which the cancellation law of addition does not hold, Bull. Amer. Math. Soc., Vol 40, 1934, $916-920$. 